\title{
Single-molecule imaging reveals Tau trapping at nanometer-sized dynamic hot spots near the plasma membrane
}

Pranesh Padmanabhan ${ }^{1, *}$, Andrew Kneynsberg ${ }^{1}$, Esteban Cruz Gonzalez ${ }^{1}$, Rumelo Amor ${ }^{2}$, JeanBaptiste Sibarita $^{3}$, Jürgen Götz, ${ }^{1, *}$

${ }^{1}$ Clem Jones Centre for Ageing Dementia Research, Queensland Brain Institute, The University of Queensland, Brisbane, Australia 4072

${ }^{2}$ Queensland Brain Institute, The University of Queensland, Brisbane, Australia 4072

${ }^{3}$ Université de Bordeaux, Interdisciplinary Institute for Neuroscience, UMR 5297, Bordeaux, France

\section{*Correspondence:}

Pranesh Padmanabhan, Jürgen Götz

Email:p.padmanabhan@uq.edu.au; j.goetz@uq.edu.au

\section{Manuscript details:}

Abstract: 150 words; Main Text: 2500 words; Figures: 5; References: 49

Supplementary Materials: Figures: 12 


\begin{abstract}
Dysregulation of Tau in Alzheimer's disease affects multiple cellular compartments and functions $^{1,2}$. Tau interacts directly with and translocates through the plasma membrane ${ }^{3-7}$, a potential site of Tau fibril and membrane pore formation ${ }^{8-10}$, but its physiological state and behavior in this compartment are unknown ${ }^{11}$. Using quantitative single-molecule imaging in live cells, we observed that Tau exhibits both confined and free diffusion near the plasma membrane. Preventing Tau/microtubule interactions either pharmacologically or biochemically increased Tau mobility; however, the heterogeneous mobility pattern persisted. Tau formed transient hot spots of $\sim 70 \mathrm{~nm}$ in radius, displaying stationary and mobile phases. Compared to the $\sim 40 \mathrm{~ms}$ dwell time of Tau on microtubules ${ }^{12}$, the hot spots were long-lasting (tens of seconds), and resistant to microtubule perturbations and cholesterol depletion. We suggest that the mobile pool acts as a reservoir supplying Tau to functional sites, with the hot spots serving as assembly sites for Tau's physiological and pathological functions.
\end{abstract}




\section{MAIN}

Tau is conventionally thought of as a protein that regulates microtubule stability. However, as an intrinsically disordered protein, Tau has remarkable structural flexibility, allowing it to interact with numerous partners and to execute multiple cellular functions ${ }^{2,13}$. Interest in the behavior of Tau at the plasma membrane was spurred firstly by the realization that, in a dementia context, toxic Tau aggregates have been reported to cross the plasma membrane and be released into the extracellular milieu, contributing to the spread of Tau pathology across interconnected brain regions ${ }^{1,14}$, and, secondly, because Tau, which lacks a classical signal peptide, undergoes unconventional secretion with an ill-defined physiological role ${ }^{6,7}$. The association of Tau with the plasma membrane can occur directly through electrostatic interactions with anionic lipids ${ }^{15,16}$ and indirectly through interactions with membrane-associated proteins, such as $\mathrm{Fyn}^{5}$, dynactin ${ }^{17}$, and annexin $\mathrm{A} 2^{18}$. Moreover, Tau interacts with microtubules and actin filaments, anchoring them to the plasma membrane ${ }^{10}$. Despite the critical importance of Tau-membrane interactions, quantifying how Tau behaves and organizes at the plasma membrane in a native cellular environment has previously proven challenging, thereby limiting our understanding of how this protein executes its function in this compartment ${ }^{11}$. Here, using single-molecule-based super-resolution microscopy, we set out to study the dynamics and nanoscale organization of Tau near the plasma membrane of live cells.

To visualize and track individual Tau molecules near the inner leaflet of the plasma membrane, we transiently expressed the most prevalent human 0N4R isoform of Tau (383 amino acids) fused carboxy-terminally to photoactivatable fluorescent protein mEos3.2 (hereafter abbreviated to Tau ${ }^{\mathrm{WT}}$-mEos3.2) in murine N2a neuroblastoma cells (Fig. 1a and Supplementary Fig. 1). We used total internal reflection fluorescence (TIRF) illumination (Fig. 1b) to perform singleparticle tracking photoactivated localization microscopy ${ }^{19}$ (sptPALM) at $50 \mathrm{~Hz}$ for a duration of 160 s. This allowed visualization of the substrate-attached cell surface comprising of the plasma membrane and its subjacent cytoskeleton with $\sim 100 \mathrm{~nm}$ axial resolution (Fig. 1c). This imaging paradigm has provided critical insights into the interactions of intracellular signaling molecules, such 


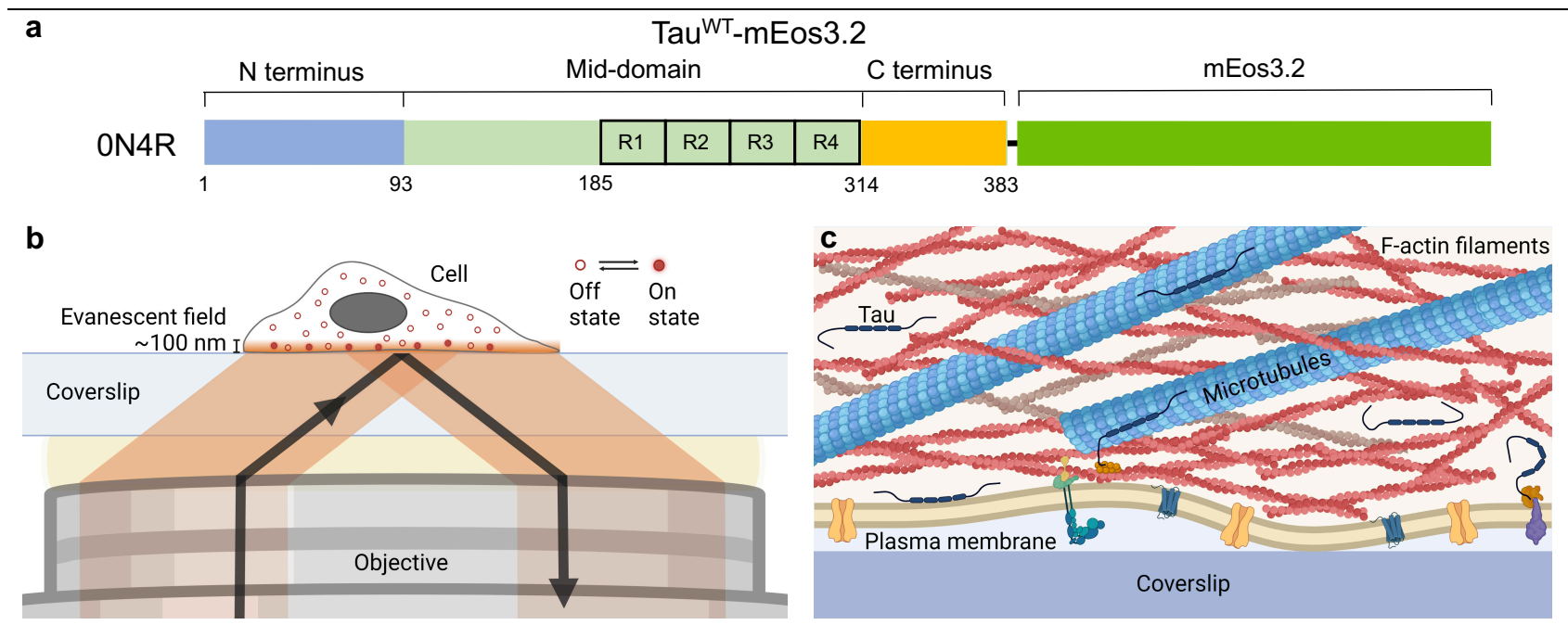

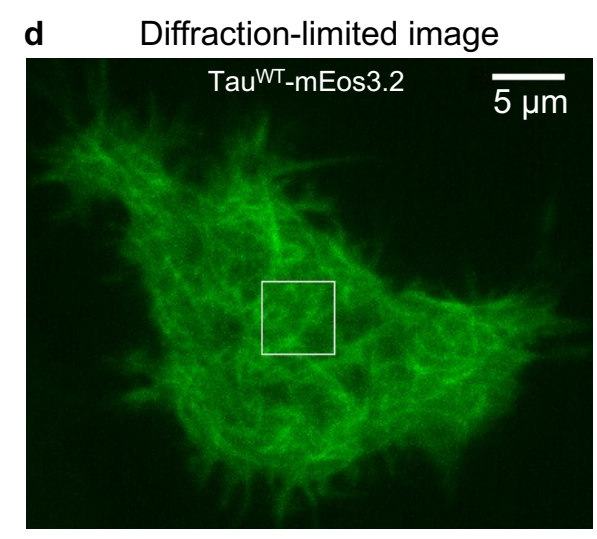

g

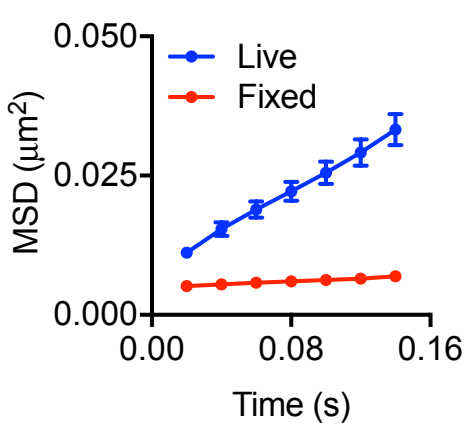

e Trajectories

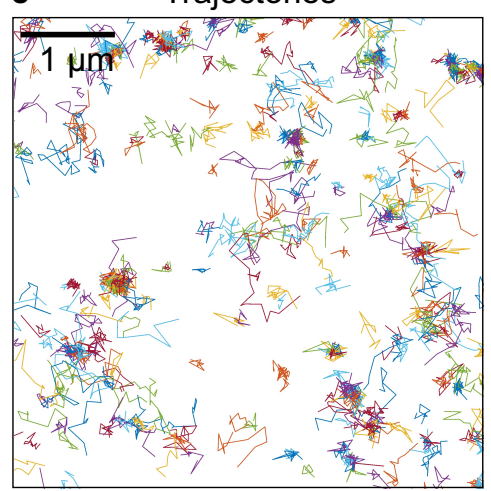

h
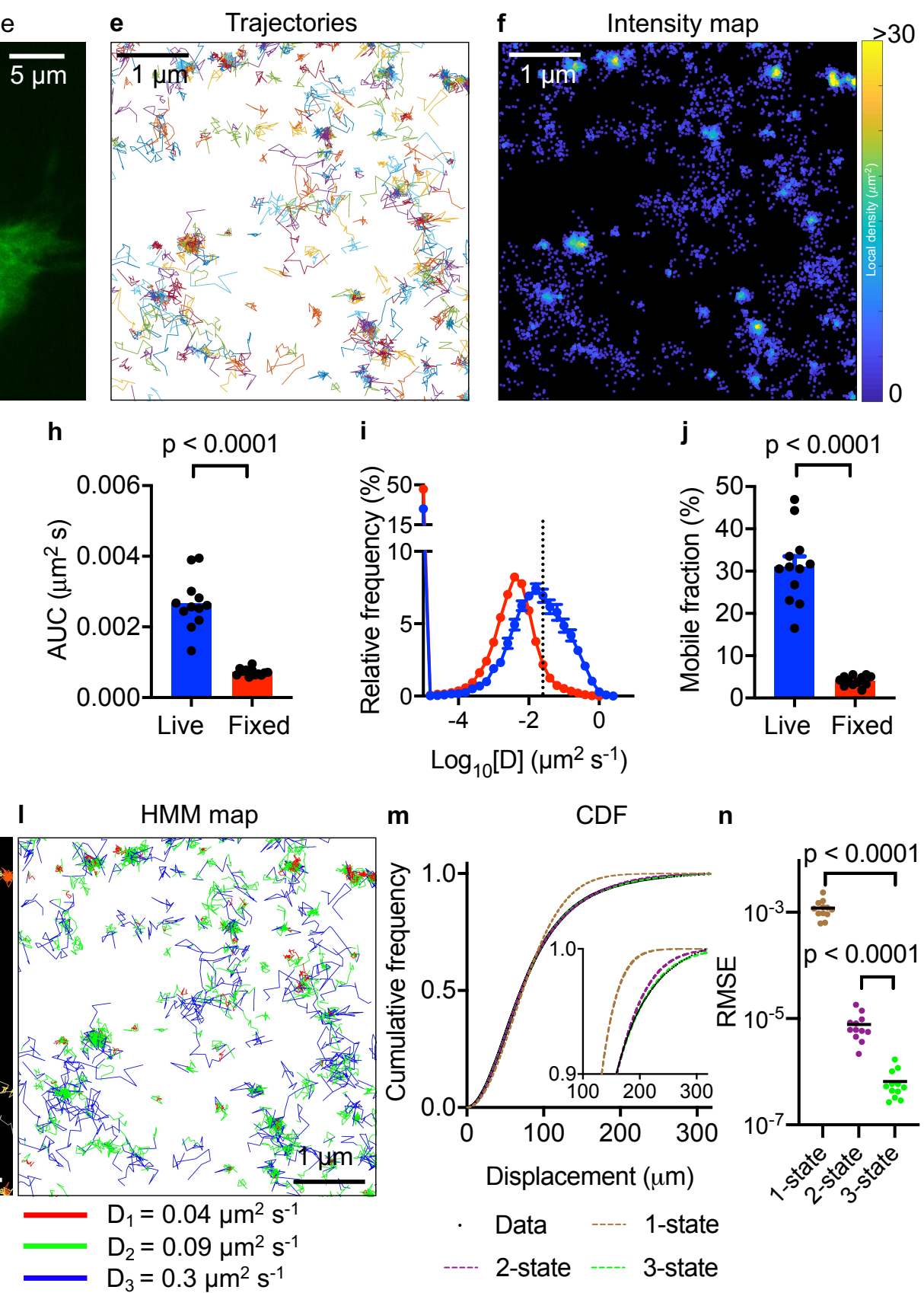

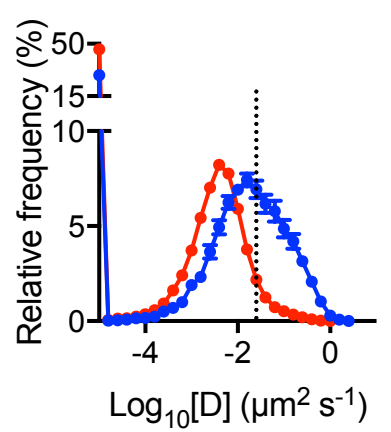

j $\quad p<0.0001$

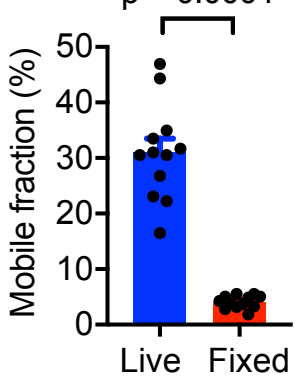

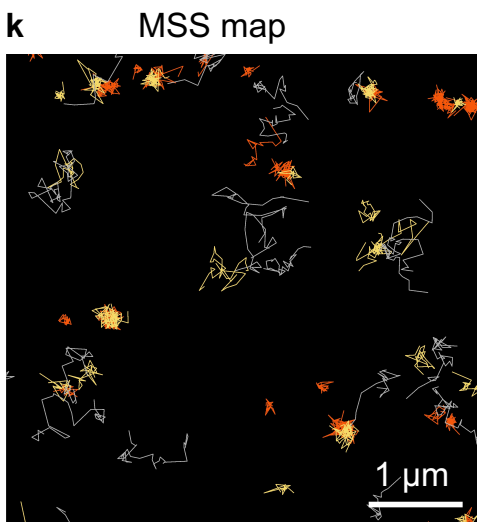

Immobile

Confined

Free m

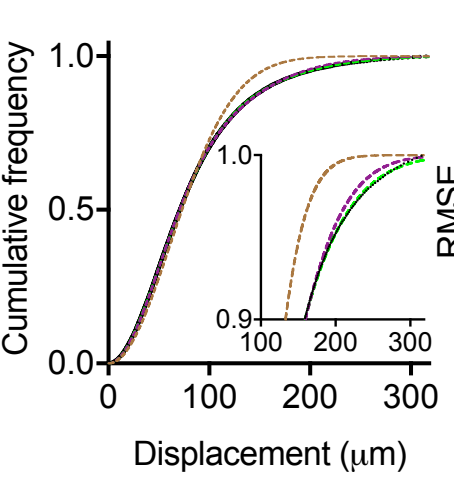

n

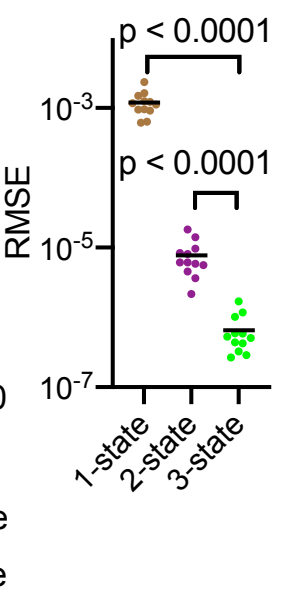

Figure 1. Tau displays heterogenous mobility patterns near the plasma membrane. a, Schematic of the most prevalent 0 N4R Tau isoform carboxy-terminally tagged with mEos3.2 (Tau ${ }^{\mathrm{WT}}$-mEos3.2). 
b, Schematic of detection of Tau ${ }^{\mathrm{WT}}$-mEos3.2 molecules in a TIRF microscopy setup. $\mathbf{c}$, Schematic of the plasma membrane and subjacent cytoskeleton captured using TIRF imaging as shown in panel $\mathbf{b}$. d, Representative diffraction-limited TIRF image of an N2a cell expressing Tau ${ }^{\mathrm{WT}}$-mEos 3.2 acquired in the green emission channel before sptPALM imaging. e,f, Maps of trajectories (e) and intensities (f) of Tau ${ }^{\mathrm{WT}}-\mathrm{mEos} 3.2$ molecules corresponding to the boxed region highlighted in D. The color bar in $\mathbf{f}$ indicates the local density of each detection computed within a radius of $30 \mathrm{~nm}$. $\mathbf{g}-\mathbf{j}$, Comparison of Tau ${ }^{\mathrm{WT}}$-mEos3.2 mobility parameters in live and fixed cells. g, Average MSD as a function of time and $\mathbf{h}$, the corresponding area under the curve. $\mathbf{i}$, The distribution of the diffusion coefficients in a semi-log plot and $\mathbf{j}$, the corresponding mobile fraction. $\mathbf{k}$, A map of trajectories annotated as immobile, confined and free state using MSS analysis. l, A map of trajectories annotated as distinct diffusive states using the 3-state hidden Markov model. m, n, Fits (m) of 1-state (red dashed line), 2state (magenta dashed line) and 3-state (green dashed line) models to the experimental data (black dotted line) and the root mean square error of all the tested models (n). Error bars, s.e.m. $\mathrm{n}=12$ live cells and 12 fixed cells. Statistical analysis was performed using the Student's $t$-test $(\mathbf{h}, \mathbf{j})$ and oneway ANOVA (n).

as $\operatorname{Rac}^{20}$ and $\mathrm{KRas}^{21}$, as well as scaffolding proteins, including caveolins ${ }^{22}$, with the plasma membrane. Capitalizing on this approach, we first captured a diffraction-limited TIRF image of $\mathrm{Tau}^{\mathrm{WT}}-\mathrm{mEos} 3.2$ before photoconversion in the green $(491 \mathrm{~nm})$ emission channel (Fig. 1d). We then recorded a time series of detections of photoconverted $\mathrm{Tau}^{\mathrm{WT}}-\mathrm{mE}$ Es 3.2 molecules near the plasma membrane in the red $(561 \mathrm{~nm})$ emission channel, with a localization precision of $\sim 47 \pm 6.5 \mathrm{~nm}$ (mean \pm s.d.) (Fig. 1e,f and Supplementary Fig. 2). This allowed us to construct 5,247 $\pm 2,373$ and $835 \pm$ 495 (mean \pm s.d.) Tau ${ }^{\mathrm{WT}}$-mEos3.2 trajectories per cell that lasted at least 8 and 20 consecutive image frames, respectively (Fig. 1e and Supplementary Fig. 3). Given that free cytosolic molecules have a large diffusion coefficient of $\sim 10-100 \mu \mathrm{m}^{2} / \mathrm{s}^{23,24}$, they would not last $\geq 8$ consecutive frames under TIRF illumination ${ }^{25}$. Thus, the detected trajectories likely represent Tau molecules trapped by plasma membrane components and the associated cytoskeleton. In line with this notion, only a few free cytosolic mEos3.2 trajectories were detected in the TIRF plane with our imaging conditions (Supplementary Fig. 3).

We next analyzed the mobility pattern of Tau by analyzing its trajectories. Typically, the interaction of a protein with itself or different plasma membrane-associated components manifests as different motion states, ranging from almost stationary to freely diffusive and directed movements ${ }^{26}$. We therefore investigated whether Tau is static or dynamic near the plasma membrane by comparing the mobility of Tau molecules in live and fixed cells. For that, we computed the average mean square 
displacement (MSD) and the frequency distribution of the instant diffusion coefficient of all trajectories from each analyzed cell (Fig. 1g-j). As expected, the slope of the average MSD curve increased, and the diffusion coefficient distribution was broader and shifted towards higher values in live cells compared to fixed cells, indicating the presence of mobile and immobile pools of Tau molecules near the plasma membrane in live cells (Fig. 1g-j and Supplementary Fig. 4). We next performed a more detailed quantification of Tau's complex motion pattern using several complementary approaches (Fig. 1k-n). First, we performed moment scaling spectrum (MSS) analysis, a method widely used to characterize motion states of receptors and transmembrane proteins $^{27}$, and estimated the slope of the MSS of Tau ${ }^{\mathrm{WT}}-\mathrm{mE}$ Es3.2 trajectories. We found that near the plasma membrane, Tau existed in three motion states: immobile, confined, and freely diffusive (Fig. 1k). The confinement radii of the immobile and confined states were $66.4 \pm 20.2 \mathrm{~nm}$ and 88.5 $\pm 38 \mathrm{~nm}$ (mean \pm s.d.), respectively (Supplementary Fig. 5a-c). We then analyzed the trajectories using hidden Markov models (HMMs) that assume molecules are switching between discrete diffusive states to estimate the associated model parameters. This analysis revealed that Tau ${ }^{\text {WT }}$ mEos3.2 molecules displayed at least three distinct diffusive states, with apparent diffusion coefficients of $0.04 \pm 0.01 \mu \mathrm{m}^{2} / \mathrm{s}, 0.1 \pm 0.01 \mu \mathrm{m}^{2} / \mathrm{s}$ and $0.29 \pm 0.02 \mu \mathrm{m}^{2} / \mathrm{s}$ (mean \pm s.d.), ranging from immobile to confined to free diffusion (Methods, Fig. 11, and Supplementary Fig. 5d-f). Finally, we fitted Brownian motion models to the cumulative frequency distribution of the frame-to-frame displacement of Tau ${ }^{\mathrm{WT}}-\mathrm{mEos} 3.2$ molecules and found that the three diffusive state model described the data well (Fig. 1m,n). Together, these results provide evidence that Tau exhibits complex diffusion patterns near the plasma membrane, possibly reflecting Tau's interactions with each other or its partners in this compartment.

Single-molecule imaging studies have revealed that receptors and signaling molecules form hot spots (a subset of which is known as nanoclusters or nanodomains) at the plasma membrane ${ }^{11}$. We therefore examined whether the observed confined diffusion is due to the similar trapping of Tau 


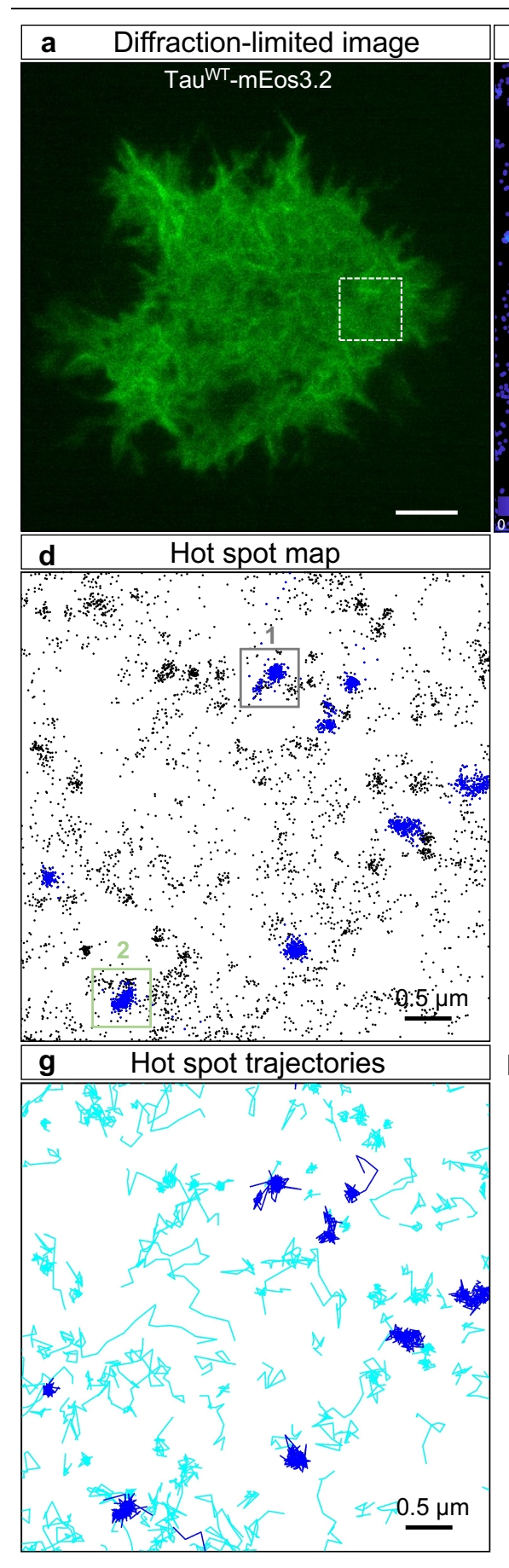

j

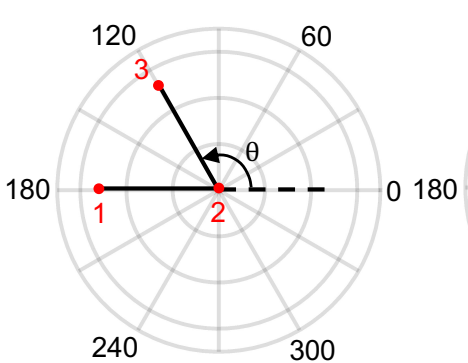

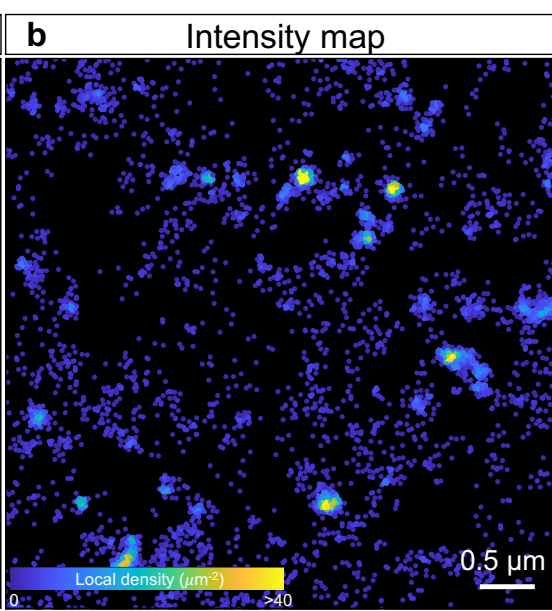

e

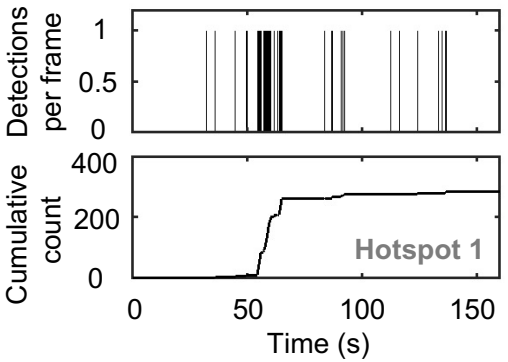

f
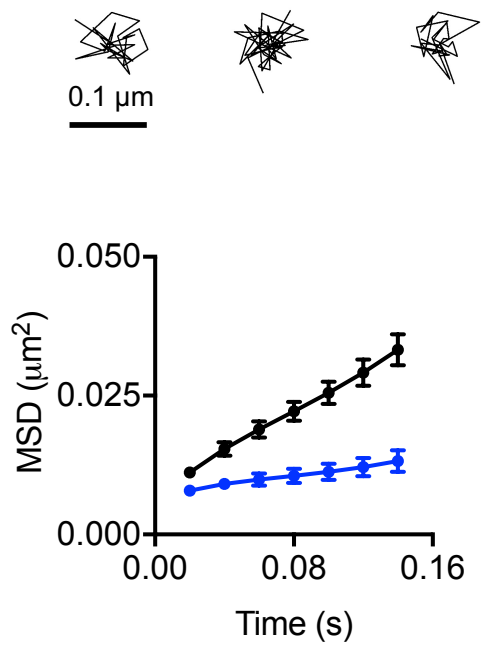

$\rightarrow$ All trajectories $\rightarrow$ Hot spot trajectories
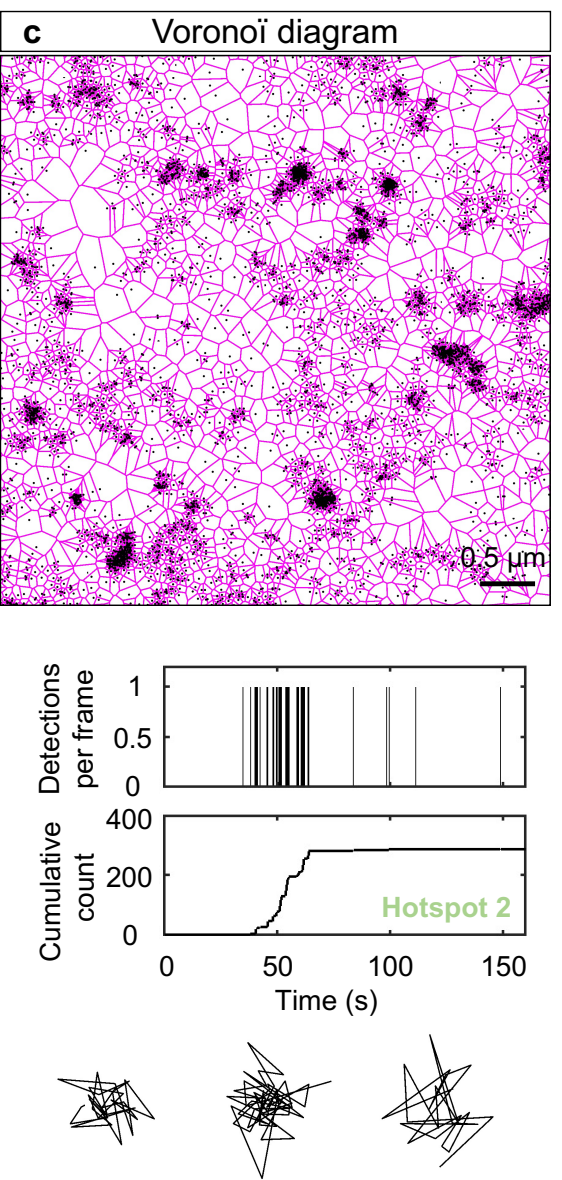

i

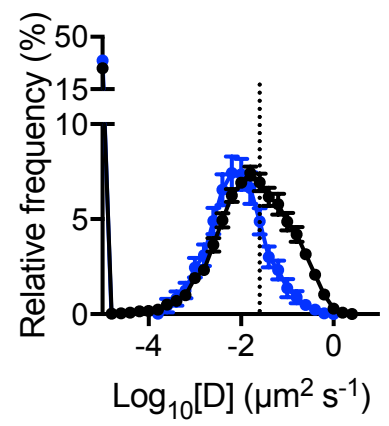

k

60

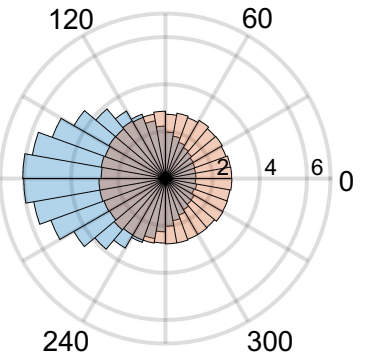

$11.9+10.9 \mathrm{~s}$

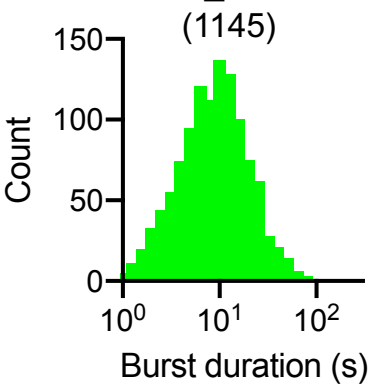

$136.1+129.2$

(1145)

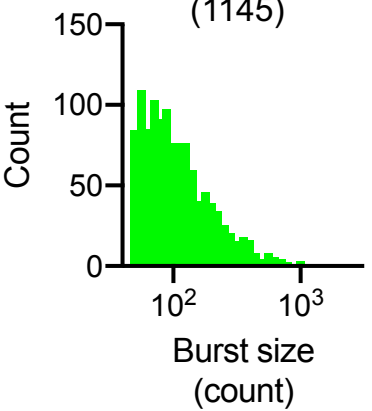

Figure 2. Tau hot spots form transiently near the plasma membrane. a, Representative diffraction-limited TIRF image of an N2a cell expressing Tau ${ }^{\mathrm{WT}}-\mathrm{mEos} 3.2$ acquired in the green channel before sptPALM imaging. b-d, Intensity map (b), Voronoï diagram (c), and hot spot map (d) 
of Tau ${ }^{\mathrm{WT}}$-mEos3.2 molecules corresponding to the boxed region highlighted in a. e, Representative time series of detections from two Tau ${ }^{\mathrm{WT}}$-mEos3.2 hot spots highlighted in d. f, Example trajectories detected within hot spots. g, Trajectories associated with hot spots are highlighted in blue and the remainder in cyan. $\mathbf{h}, \mathbf{i}$, Comparison of mobility parameters of TauWT-mEos 3.2 molecules associated with hot spots and all the trajectories. Error bars, s.e.m. j, Angular distribution of the angle $(\theta)$ between the vectors of two successive translocation steps of molecules within hot spots (light blue) compared with that of simulated molecules undergoing Brownian motion (light red). $\theta$ is the angle between the vector joining the $1^{\text {st }}$ and $2^{\text {nd }}$ positions and the vector connecting the $2^{\text {nd }}$ and $3^{\text {rd }}$ positions. $\mathbf{k}, \mathbf{l}$, The distributions of burst duration (k) and size (l) of hot spots. The mean \pm s.d. are shown together with the number of hot spots analyzed in brackets $(n=12$ cells $)$.

in hot spots. Indeed, we found signatures of Tau hot spots with high localization density in the intensity maps that were not detectable in the diffraction-limited TIRF image (Fig. 2a,b). We quantitatively characterized these regions based on both spatial and temporal correlations between individual detections associated with the hot spots as follows. Tessellation-based spatial segmentation algorithms have been widely employed to quantify protein clusters from the sptPALM data ${ }^{28,29}$. Using this approach, we first generated a Voronoï diagram of all localizations, with polygons centered on each single-molecule localization in cells (Fig. 2c). We then identified the cell contour and the potential locations of Tau hot spots based on the normalized localization detection parameters (Methods, Fig. 2d). Next, to characterize their temporal dynamics, we performed time-correlated PALM (tcPALM) analysis, which has previously revealed transient clustering of RNA polymerase $^{30,31}$ and membrane receptors ${ }^{22}$. Here, we computed the time series of molecular detections of individual hot spots and found that the detections were not uniformly distributed but were correlated and clustered in time, indicating the transient nature of these hot spots near the plasma membrane (Fig. 2e). This effect was more apparent in the cumulative detections of molecules, where we observed sudden changes in the slope of detection frequency, marking the duration during which we could detect the hot spots (Fig. 2e). We also found that the trajectories within the hot spots were highly confined within a radius of $71.8 \pm 31 \mathrm{~nm}$ (mean \pm s.d.) (Fig. 2f, Supplementary Fig. 5g). Furthermore, the mobility of Tau ${ }^{\mathrm{WT}}-\mathrm{mE}$ Es 3.2 molecules in these hot spots was significantly lower than their overall mobility (Fig. 2g-i). To better characterize this confinement, we computed the angles between two successive translocations from three consecutive points and the angular distribution of Tau molecules within the hot spots (Fig. 2j) that together allows for understanding the 


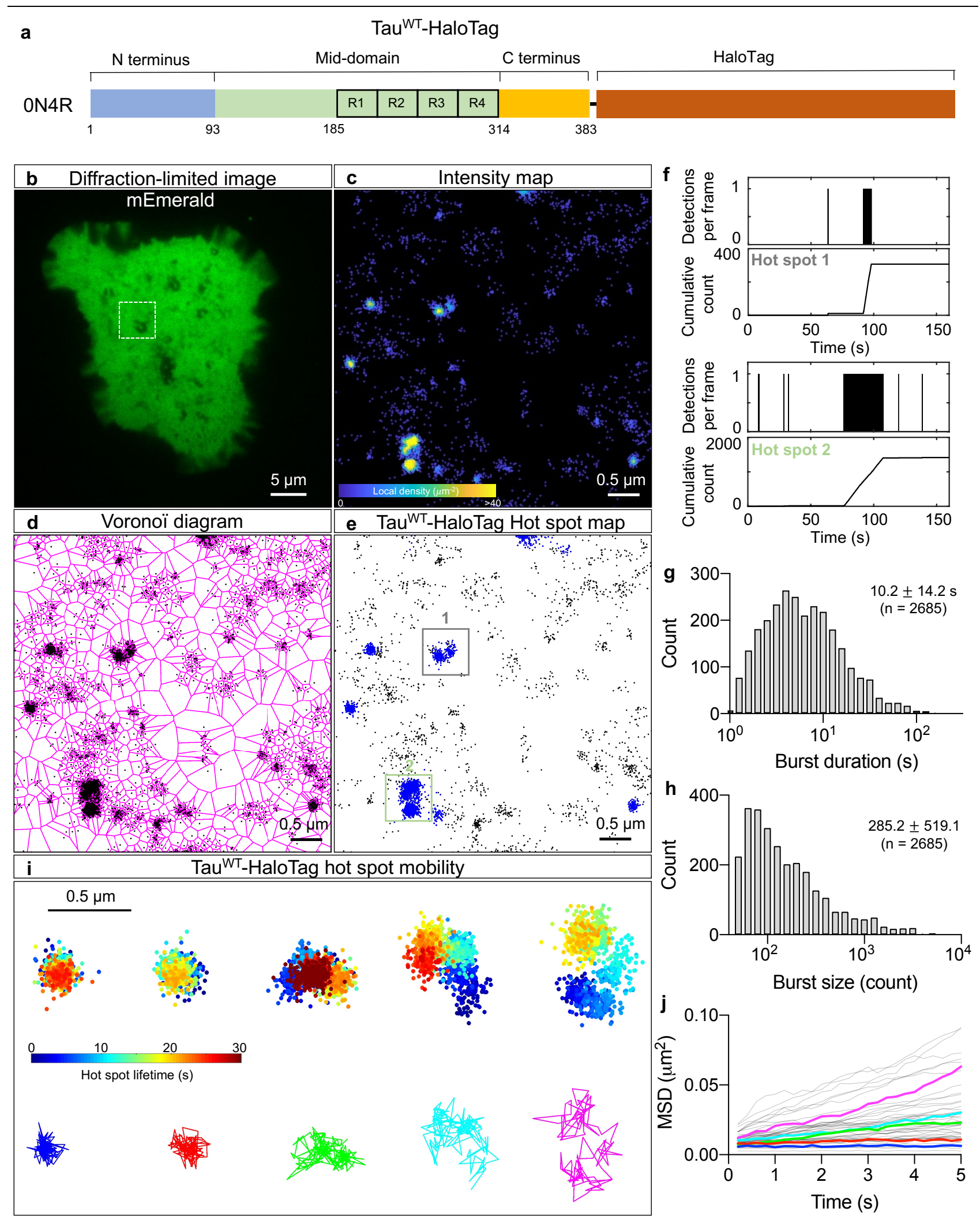

Figure 3. Tau hot spots display distinct motion near the plasma membrane. a, Schematic of the 0N4R Tau isoform carboxy-terminally tagged with Halo-Tag (Tau ${ }^{\text {WT }}$-HaloTag). b, Representative diffraction-limited image of an $\mathrm{N} 2 \mathrm{a}$ cell expressing mEmerald acquired in the green emission channel before single-molecule imaging of Tau ${ }^{\mathrm{WT}}$-HaloTag in the far-red channel. c-e, Intensity map (c), Voronoï diagram (d), and hot spot map (e) of Tau ${ }^{\mathrm{WT}}$-HaloTag molecules corresponding to the boxed region highlighted in a. f, Representative time series of detections from Tau ${ }^{\mathrm{WT}}$-HaloTag hot spots highlighted in d. $\mathbf{g}, \mathbf{h}$, Distributions of burst duration and size. The mean \pm s.d. are shown together 
with the number of hot spots analyzed in brackets $(n=11$ cells). $\mathbf{i}$, Examples of hot spots that are immobile (left) and mobile (right). Top, all the localization of hot spots color-coded based on time since hot spot detection. Bottom, trajectories of hot spots constructed by sampling localization belonging to hot spots at $200 \mathrm{~ms}$ interval. $\mathbf{j}$, MSDs of Tau ${ }^{\text {WT }}$. HaloTag hot spots with colored curves representing hot spots highlighted in $\mathbf{i}$.

geometry of the local environment of the molecule ${ }^{32}$. The angular distribution of Tau within the hotspots was shifted towards $180^{\circ}$ and significantly different from what is expected from random motion (Fig. 2j) and consistent with motion within a confined space. We next estimated the lifetime of each burst (burst duration) and the number of detections per burst (burst size). The average burst duration and size of the Tau hot spots were $11.9 \pm 10.9 \mathrm{~s}$ and $136.1 \pm 129.2$ detection counts (mean \pm s.d.), respectively (Fig. 2k,1). Finally, we found that TauWT-mEos3.2 molecules also formed hot spots and displayed heterogeneous mobility patterns in HEK293T cells (Supplementary Fig. 6) similar to those observed in N2a cells, indicating that the dynamic behavior of Tau is conserved across cell types.

To ascertain that the Tau hotspots were not an artifact due to the presence of the mEos 3.2 tag, we performed single-molecule imaging of $\mathrm{Tau}^{\mathrm{WT}}$ fused with the HaloTag in N2a cells incubated with a HaloTag ligand (Janelia Fluor 646). At a 100 pM concentration of the Halo ligand, we only obtained single-molecule signals in N2a cells transfected with Tau ${ }^{\mathrm{WT}}$-HaloTag but not in cells transfected with either the free HaloTag or Tau ${ }^{\text {WT }}$-FLAG-Tag (Supplementary Fig. 7). By combining Voronoï tessellation-based spatial segmentation and tcPALM analyses, we observed that Tau ${ }^{\text {WT }}$-HaloTag molecules also formed hot spots near the plasma membrane (Fig. 3a-e). The average burst duration and size of Tau ${ }^{\text {WT }}$-HaloTag hot spots were $10.2 \pm 14.2 \mathrm{~s}$ and $285.2 \pm 519.1$ detection counts (mean \pm s.d.), respectively (Fig. 3f-h). Membrane protein clusters often exhibit lateral mobility with functional consequences $^{33}$. For example, DC-SIGN and LAT cluster mobility are linked to virus internalization and $\mathrm{T}$ cell signaling, respectively ${ }^{33}$. We therefore wondered whether Tau hot spots can also display movements. To test this, we constructed trajectories of Tau ${ }^{\text {WT }}$-HaloTag hot spots at $0.2 \mathrm{~s}$ intervals over their lifetime (Fig. 3i). We then quantified the MSD curves of hot spot trajectories and found that some hot spots were stationary whereas others were mobile (Fig. 3j). Overall, these results show 


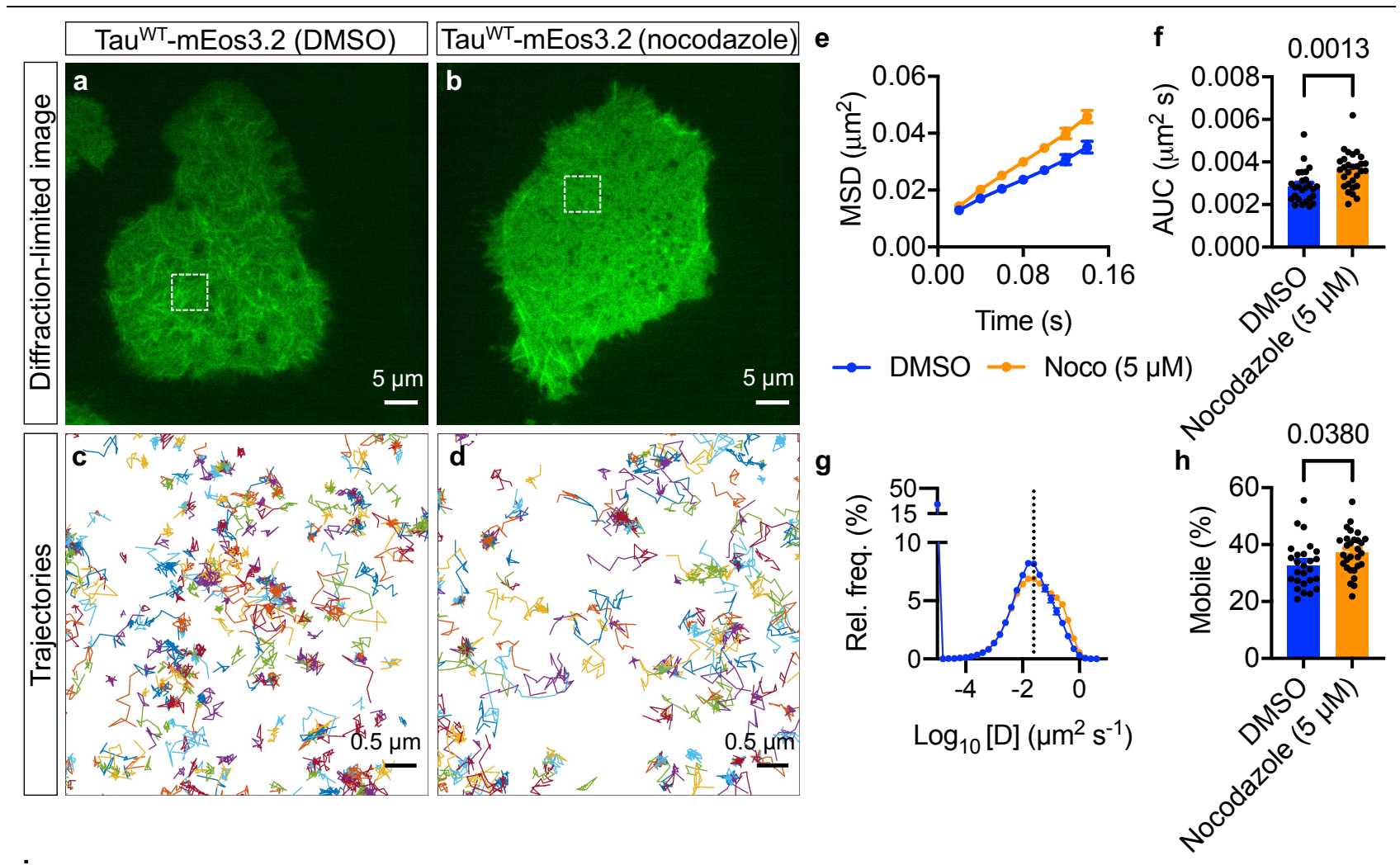

Tau ${ }^{\text {S262E/S356E-mEos3.2 }}$
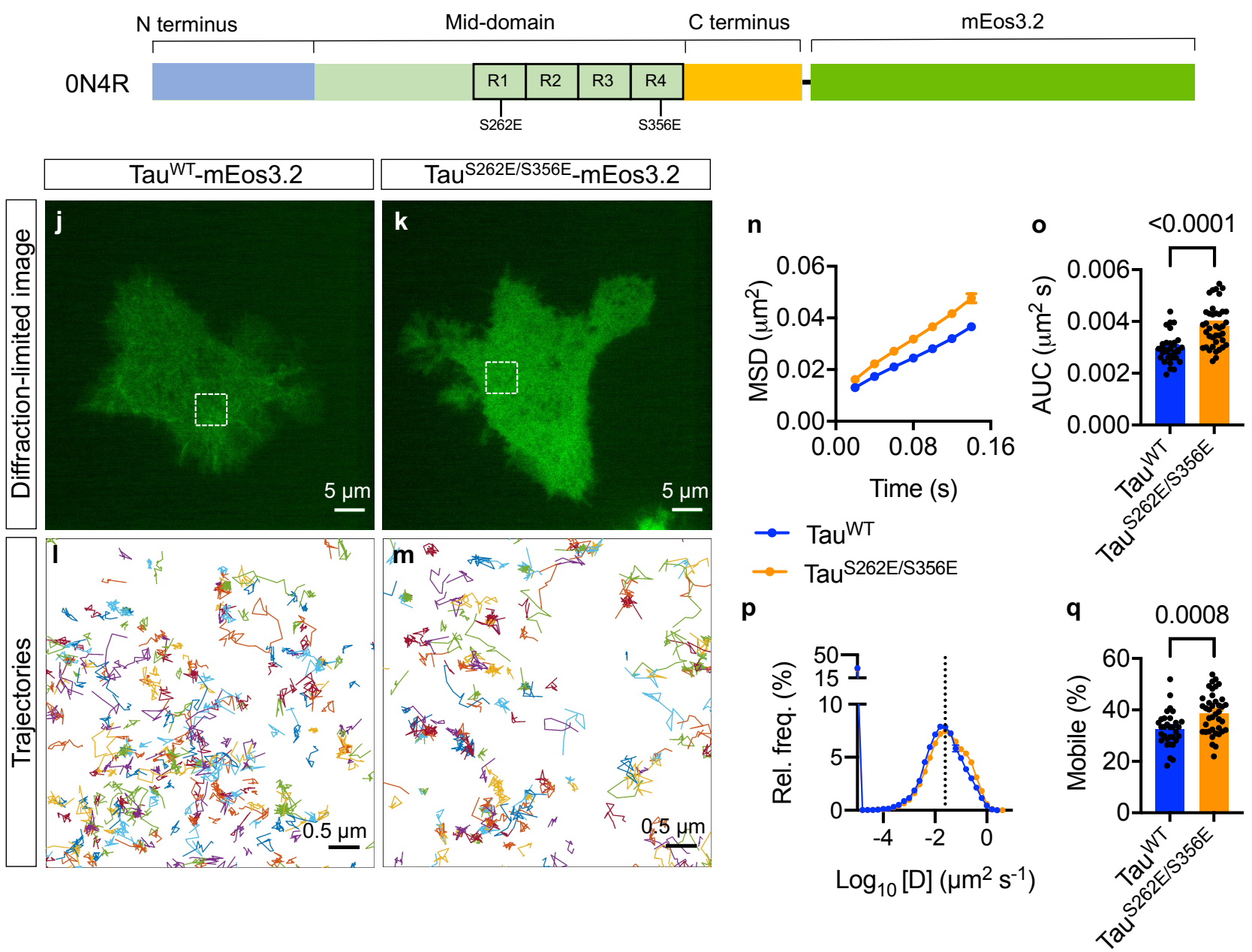

Figure 4. Preventing Tau-microtubule interactions increases Tau's mobility near the plasma membrane. a, b, Representative diffraction-limited TIRF image of N2a cells expressing Tau ${ }^{\mathrm{WT}}$ mEos3.2 treated with either DMSO (a) or $5 \mu \mathrm{M}$ nocodazole (b) acquired before sptPALM imaging. 
Scale bar, $5 \mu \mathrm{m}$. c, $\mathbf{d}$, Maps of trajectories of Tau ${ }^{\mathrm{WT}}-\mathrm{mEos} 3.2$ molecules corresponding to the boxed region highlighted in $\mathbf{a}$ and $\mathbf{b}$. e-h, Comparison of $\mathrm{Tau}^{\mathrm{WT}}$-mEos3.2 mobility parameters in DMSOand nocodazole-treated live cells. e,f, Average MSD as a function of time (e) and the corresponding area under the curve (f). $\mathbf{g}, \mathbf{h}$, The distribution of diffusion coefficient $(\mathbf{g})$ shown in semi-log plot and the corresponding mobile fractions $(\mathbf{h}) . \mathrm{n}=26$ DMSO-treated and 29 nocodazole-treated cells from 3 independent experiments. i, Schematic of 0N4R Tau tagged with mEos3.2 at the C-terminus with S262E/S356E mutations to disrupt microtubule binding. j, k, Representative diffraction-limited TIRF image of N2a cell expressing Tau ${ }^{\mathrm{WT}}-\mathrm{mEos} 3.2(\mathbf{j})$ and $\mathrm{Tau}^{\mathrm{S} 262 \mathrm{E} / \mathrm{S} 356 \mathrm{E}}-\mathrm{mEos} 3.2(\mathbf{k})$. l, m, Maps of trajectories of Tau ${ }^{\mathrm{WT}}-\mathrm{mEos} 3.2$ and $\mathrm{Tau}^{\mathrm{S} 262 \mathrm{E} / \mathrm{S} 356 \mathrm{E}}-\mathrm{mEos} 3.2$ molecules corresponding to the box region highlighted in $\mathbf{l}$ and $\mathbf{m}$, respectively. $\mathbf{n}-\mathbf{q}$, Comparison of Tau ${ }^{\mathrm{WT}}-\mathrm{mE} \cos 3.2$ and $\mathrm{Tau}^{\mathrm{S} 262 \mathrm{E} / \mathrm{S} 356 \mathrm{E}}-\mathrm{mE}$ os 3.2 mobility parameters in live cells. $\mathbf{n}, \mathbf{0}$, Average MSD as a function of time (n) and corresponding AUC (o). p-q, The distribution of diffusion coefficient (p) shown in semi-log plot and the corresponding immobile fraction $(\mathbf{q}) \cdot \mathrm{n}=32$ cells expressing Tau ${ }^{\mathrm{WT}}-\mathrm{mEos} 3.2$ and 39 cells expressing $\mathrm{Tau}^{\mathrm{S} 262 \mathrm{E} / \mathrm{S} 356 \mathrm{E}}-\mathrm{mE}$ os 3.2 from 4 independent experiments. Statistical analysis was performed using the Student's $t$-test.

that Tau forms hot spots that are dynamic at the cell surface.

Given that microtubules are a major binding partner of Tau and that microtubules localize to and interact with the plasma membrane, we next asked whether the mobility pattern of Tau is affected by the availability of microtubules. Indeed, a diffraction-limited TIRF image of the Tau ${ }^{\mathrm{WT}}-\mathrm{mEos} 3.2$ expressing cells readily revealed microtubule-like structures indicative of the association of Tau with the microtubules (Fig. 4a). To assess the effect microtubules have on Tau's mobility, we treated the $\mathrm{N} 2 \mathrm{a}$ cells with either $5 \mu \mathrm{M}$ of the microtubule-destabilizing agent nocodazole or DMSO (control) for up to 4 hours. As expected, in the diffraction-limited TIRF images, the density of microtubule filament-like structures visualized by $\mathrm{Tau}^{\mathrm{WT}}-\mathrm{mE}$ - 3.2 molecules was markedly reduced in nocodazole-treated compared to DMSO-treated live cells (Fig. 4a,b and Supplementary Fig. 8a). A similar effect was observed when imaging the same cell before and after nocodazole treatment (Supplementary Fig. 8b). We also saw a similar decrease in microtubule filaments in fixed, nocodazole-treated N2a cells stained with an anti-tubulin antibody (Supplementary Fig. 9). Consistent with what has been previously reported ${ }^{34}$, we did observe a few nocodazole-resistant microtubules. Remarkably, the trajectory maps showed that Tau molecules could be detected in both DMSO- and nocodazole-treated cells (Fig. 4c,d). We further found that the mobility of Tau was higher in the nocodazole-treated compared to control cells, as assessed by changes in the average MSD (Fig. 4e), the area under the curve (Fig. 4f), the frequency distribution of diffusion coefficients 

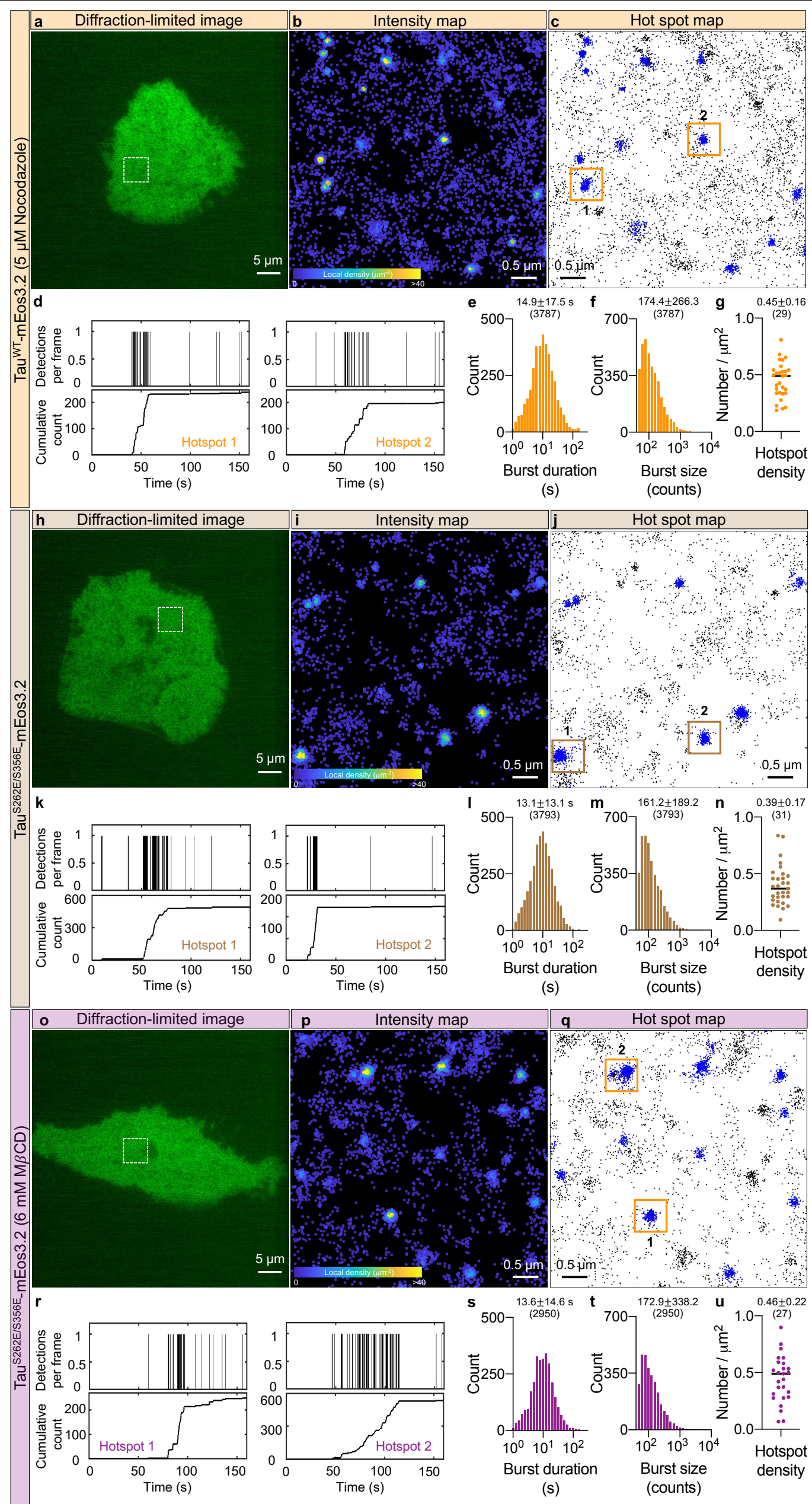
Figure 5. Tau hot spots near the plasma membrane are resistant to both microtubule perturbation and cholesterol depletion. a, Representative diffraction-limited TIRF image of an N2a cell expressing Tau ${ }^{\mathrm{WT}}$-mEos3.2 in nocodazole-treated live cells. b, c, Maps of intensities (b) and hot spots $(\mathbf{c})$ of $\mathrm{Tau}^{\mathrm{WT}}$-mEos 3.2 corresponding to the boxed region highlighted in $\mathbf{a}$. d, Representative time series of detections from Tau ${ }^{\mathrm{WT}}$-mEos3.2 hot spots highlighted in $\mathbf{d}$. e, f, Distribution of burst duration and size. $\mathbf{g}$, Variations in the density of detected hot spots across cells. $n=29$ nocodazoletreated cells from 3 independent experiments. $\mathbf{h}$, Representative diffraction-limited TIRF image of an $\mathrm{N} 2 \mathrm{a}$ cell expressing Tau ${ }^{\mathrm{S} 262 \mathrm{E} / \mathrm{S} 356 \mathrm{E}}$-mEos3.2 in live untreated cells. Scale bar, $5 \mu \mathrm{m}$. i, j, Maps of intensities (i) and hot spots $(\mathbf{j})$ of $\mathrm{Tau}^{\mathrm{S} 262 \mathrm{E} / \mathrm{S} 356 \mathrm{E}}-\mathrm{mEos} 3.2$ corresponding to the boxed region highlighted in $\mathbf{h}$. $\mathbf{k}$, Representative time series of detections from $\mathrm{Tau}^{\mathrm{WT}}-\mathrm{mEos} 3.2$ hot spots highlighted in $\mathbf{h}$. $\mathbf{l}, \mathbf{m}$, Distributions of burst duration and burst size. $\mathbf{n}$, Variations in the density of detected hot spots across cells. $n=39$ cells expressing Tau ${ }^{\mathrm{S} 262 \mathrm{E} / \mathrm{S} 356 \mathrm{E}}-\mathrm{mE}$ - 3.2 from 4 independent experiments. o, Representative diffraction-limited TIRF image of an N2a cell expressing $\mathrm{Tau}^{\mathrm{S} 262 \mathrm{E} / \mathrm{S} 356 \mathrm{E}}-\mathrm{mE}$ os 3.2 in live cholesterol-depleted cells. p, q, Maps of intensities (p) and hot spots (q) of $\mathrm{Tau}^{\mathrm{S} 262 \mathrm{E} / \mathrm{S} 356 \mathrm{E}}-\mathrm{mEos} 3.2$ corresponding to the boxed region highlighted in $\mathbf{o}$. $\mathbf{r}$, Representative time series of detections from $\mathrm{Tau}^{\mathrm{WT}}-\mathrm{mEos} 3.2$ hot spots highlighted in q. $\mathbf{s}, \mathbf{t}$, The distributions of burst duration and burst size. $\mathbf{u}$, Variations in the density of detected hot spots across cells. $\mathrm{n}=27$ cells expressing Tau ${ }^{\mathrm{S} 262 \mathrm{E} / \mathrm{S} 356 \mathrm{E}}-\mathrm{mEos} 3.2$ treated with $6 \mathrm{mM}$ M $\beta C D$ from 4 independent experiments. In $\mathbf{e}, \mathbf{f}, \mathbf{l}, \mathbf{m}, \mathbf{s}, \mathbf{t}$, The mean \pm s.d. are shown together with the number of hot spots analyzed in brackets. In $\mathbf{g}, \mathbf{n}, \mathbf{u}$, The mean \pm s.d. are shown together with the number of cells analyzed in brackets.

(Fig. 4g), and the percentage of the mobile fraction (Fig. 4h).

To further validate this effect, we generated a pseudophosphorylated mutant Tau tagged with mEos3.2 by introducing two serine to glutamate mutations at positions 262 and 356 in the microtubule-binding domain $\left(\mathrm{Tau}^{\mathrm{S} 262 \mathrm{E} / \mathrm{S} 356 \mathrm{E}}-\mathrm{mE} \mathrm{s} 3.2\right)$ that reduce Tau's binding affinity to microtubules $^{35}$ (Fig. 4i). As expected, the microtubule filament-like structures were barely visible in $\mathrm{Tau}^{\mathrm{S} 262 \mathrm{E} / \mathrm{S} 356 \mathrm{E}}$-mEos3.2-expressing cells compared to those expressing Tau ${ }^{\mathrm{WT}}-\mathrm{mE}$ - 3.2 (Fig. $4 \mathrm{j}, \mathrm{k}$ and Supplementary Fig. 10), indicating a reduced association of the mutant Tau with the microtubules. Yet, we could readily detect and track a similar number of molecules in both Tau ${ }^{\mathrm{WT}}$-mEos3.2- and $\mathrm{Tau}^{\mathrm{S} 262 \mathrm{E} / \mathrm{S} 356 \mathrm{E}}$-mEos3.2-expressing cells (Fig. 41-m and Supplementary Fig. 11a). Consistent with our nocodazole experiments, we found that $\mathrm{Tau}^{\mathrm{S} 262 \mathrm{E} / \mathrm{S} 356 \mathrm{E}_{-}}-\mathrm{mE} \mathrm{Es} 3.2$ had higher mobility than Tau ${ }^{\mathrm{WT}}$ mEos3.2 (Fig. 4n-q). For both conditions, HMM analysis revealed that Tau molecules displayed at least three distinct diffusive states (Supplementary Fig. 11). Together, these results indicate that the mobility of Tau increases when it is released from microtubules and that the protein exhibits heterogeneous mobility patterns even when the Tau/microtubule interactions are prevented. 
We next investigated the effect of microtubule perturbations on the dynamics of Tau hot spots. We were able to detect transient hot spots of Tau ${ }^{\mathrm{WT}}$-mEos3.2 in nocodazole-treated cells (Fig. 5a-g) and of Tau ${ }^{\mathrm{S} 262 \mathrm{E} / \mathrm{S} 356 \mathrm{E}}-\mathrm{mE} 0 \mathrm{~s} 3.2$ in untreated cells (Fig. 5h-n). In microtubule-perturbed conditions, the burst duration and size of the Tau hot spots were $\sim 13-15$ s and $\sim 160-180$ detection counts, respectively, and the density of the hot spots was $\sim 0.4-0.5$ spots $/ \mu \mathrm{m}^{2}$, comparable to the values obtained for Tau ${ }^{\mathrm{WT}}-\mathrm{mE}$ Es 3.2 in untreated cells. Previous studies have described cholesteroldependent and -independent mechanisms of protein clustering at the plasma membrane ${ }^{36}$, and cholesterol depletion has been shown to decrease the unconventional release of Tau through the plasma membrane in $\mathrm{N} 2 \mathrm{a}$ cells $\mathrm{s}^{7}$. We therefore examined the effect of cholesterol depletion on the dynamics of Tau hot spots by treating Tau ${ }^{\mathrm{S} 262 \mathrm{E} / \mathrm{S} 356 \mathrm{E}}-\mathrm{mE}$-s3 3.2 -expressing N2a cells with $6 \mathrm{mM}$ methyl- $\beta$-cyclodextrin (M $\beta \mathrm{CD}$ ), a concentration known to strongly deplete cholesterol. Interestingly, there was no apparent effect of cholesterol depletion on the dynamics of Tau hot spots. The burst duration, burst size and density of Tau hot spots were $13.6 \pm 14.6 \mathrm{~s}, 172.9 \pm 338.2$ detection counts, and $0.46 \pm 0.22$ spots $/ \mu \mathrm{m}^{2}$ (mean \pm s.d.), respectively (Fig. 5o-u). Together, these findings that Tau spots are resistant to microtubule perturbations and cholesterol depletion.

In summary, our study sheds new light on the complex, spatiotemporal organization of Tau near the plasma membrane. We revealed that Tau exists in distinct motion states in this region: (i) it may be immobile and trapped inside hot spots, (ii) microtubules may transiently confine it, or (iii) it may undergo free diffusion along the plasma membrane. Cytosolic proteins use different routes, varying in their time spent in different mobility states, to reach their target sites at the plasma membrane. A case in point is the molecular dynamics linked to focal adhesion (FA) zones. For instance, some proteins, such as Rac1 and kindlin, are targeted to the membrane where they can undergo free diffusion before being recruited to and confined at these zones ${ }^{37,38}$. By contrast, proteins such as talin and PIX are directly recruited from the cytoplasm to these zones and become confined $^{37,38}$. Why proteins choose different recruitment pathways appears to be dictated by their functions and is still a matter of intense investigation. Given that a significant proportion of Tau 
molecules freely diffuses near the plasma membrane, we speculate that free diffusion may allow Tau to explore the membrane efficiently and that this pool serves as a reservoir to supply Tau to its multiple interactors at the plasma membrane. Consistent with this notion, blocking Tau's interactions with microtubules increased the mobile pool of Tau.

Protein clustering has emerged as a dominant feature of membrane organization ${ }^{11,39}$, with the molecular characteristics and functional significance of protein clusters at the membrane slowly emerging $^{26}$. In our study, we observed transient hot spots of Tau near the plasma membrane that resemble membrane receptor clusters ${ }^{22}$ and RNA polymerase and mediator complex condensates ${ }^{30,31}$. What are these Tau hot spots and which functions do they exert? One possibility is that the preexisting assemblies of Tau's interaction partner(s) such as Fyn and annexin may serve as sites to capture and trap Tau molecules as they pass. Alternatively, Tau may itself form assemblies near the plasma membrane, influencing biochemical reactions in the local environment. Notably, the burst duration of hot spots varied by up to two orders of magnitude in all tested conditions, indicating substantial variability in the kinetics of hot spots and suggesting that multiple mechanisms determining hot spot formation and their stability may be at play, with some assemblies destined to be extracellularly released and others to remain and undertake membrane functions. Remarkably, the lifetime of a hot spot far exceeds (by two orders of magnitude) the reported $\sim 40 \mathrm{~ms}$ dwell time of Tau on microtubules ${ }^{12}$, hinting that the interactions of Tau with different partners lead to different Tau behaviors and potentially different biological outcomes. It is tempting to speculate that it is not only the dynamics of individual hot spots but also the coordination of multiple hot spots at the plasma membrane that determines Tau's membrane functions. Whether Tau hot spots contribute to membrane insertion and fibrillization in pathological conditions such as Alzheimer's disease remains to be determined. 


\section{ACKNOWLEDGMENTS}

We thank Rowan Tweedale for critical reading of the manuscript. We acknowledge Corey Butler and Adel Kechkar for their contributions in the development of PALM-Tracer. The imaging was performed at the Queensland Brain Institute Advanced Microscopy Facility, supported by the Australian Government through the Australian Research Council LIEF grant (LE130100078). We acknowledge support by the Estate of Dr. Clem Jones, the State Government of Queensland (DSITI, Department of Science, Information Technology and Innovation), and the National Health and Medical Research Council of Australia (GNT1176326, GNT1147569, and GA39196) to J.G.

\section{COMPETING INTERESTS}

The authors declare that no conflict of interest exist.

\section{METHODS}

\section{Cell culture and transfection}

N2a mouse neuroblastoma cells were maintained in Dulbecco's minimum essential medium (ThermoFisher; Catalog No. 11965-092) supplemented with 10\% foetal bovine serum (Bovogen; Catalog No. SFBS-F) and $50 \mathrm{U} / \mathrm{ml}$ penicillin/streptomycin (Gibco; Catalog No. 15140-122). Cells were grown at $37^{\circ} \mathrm{C}$ in $5 \% \mathrm{CO}_{2} .250,000$ cells per well were plated in 12 -well plates for $18 \mathrm{~h}$ before transfection. Lipofectamine ${ }^{\mathrm{TM}}$ LTX and Plus reagent (Invitrogen; Catalog No. 15338030) were used for cell transfections as per the manufacturer's instructions. For imaging, the cells were dissociated with Trypsin-EDTA (0.25\%; Catalog No. 25200-056), centrifuged, and $~ 32,000$ cells were replated on poly-D-lysine-coated $35 \mathrm{~mm}$ glass-bottom culture dishes (Ibidi) for one hour. The media was then full changed, and cells were imaged after 16 to $40 \mathrm{~h}$. 


\section{Plasmids}

Mammalian expression plasmids were made using human tau cDNA for isoform 0N4R and the human cytomegalovirus (CMV) promoter. Fusion proteins were created with a C-terminal tag of mEos3.2, HaloTag or FLAG-tag (DYKDDDDK). The plasmid mEos3.2-C1 was a gift from Michael Davidson and Tao Xu (RRID:Addgene_54550) ${ }^{40}$. Pseudo-phosphorylated Tau at Ser262 and Ser356 was made by substitution of serine by glutamic acid. Plasmids for control expression of tagged proteins were created by deletion of the tau encoding sequence.

\section{Western blot analysis}

$\mathrm{N} 2 \mathrm{a}$ cells were collected for expression validation from the 12-well plate at $24 \mathrm{~h}$ post transfection in RIPA buffer (Cell Signalling Technologies; Catalog No. 9806) in the presence of phosphatase inhibitor (Roche; Catalog No. 04906837001) and protease inhibitor (Roche; Catalog No. 04693159001). Samples were diluted in Laemmli buffer, sonicated and heated to $95^{\circ} \mathrm{C}$ for 10 minutes, then separated using SDS-PAGE on 4-20\% Criterion TGX (BioRad; Catalog Nos. 5671084 and 5671085) gradient gels at $250 \mathrm{~V}$. The samples were transferred to nitrocellulose membranes (Merck; Catalog No. HATF00010) for 50 minutes at $400 \mathrm{~mA}$. The membranes were blocked in TBS containing Odyssey Blocking Buffer (LI-COR; Catalog No.927-50000) and incubated in primary antibodies (Tau5 1:5,000) overnight at $4{ }^{\circ} \mathrm{C}$ followed by incubation with the secondary antibody for one hour at RT. Membranes were imaged on the LI-COR Odyssey scanner using the Image Studio software (LI-COR; Catalog Nos. 926-32211 and 926-68070).

\section{TIRF microscopy and single-molecule imaging}

For live-cell TIRF microscopy, transfected cells were imaged using an iLas ${ }^{2}$ azimuthal TIRF illumination system (Roper Scientific) mounted on a Nikon Ti-E inverted microscope, with a 100x/1.49 NA oil-immersion TIRF objective (CFI Apochromat, Nikon) and Evolve 512 Delta EMCCD cameras (Teledyne Photometrics). Image acquisition was performed using MetaMorph 
(version 7.10.1.161, Molecular Devices). Single-molecule imaging of Tau tagged with mEos3.2 was performed at $50 \mathrm{~Hz}$ to record 8,000 frames per cell at $37^{\circ} \mathrm{C}$. Cells were washed and incubated with buffer A (145 mM NaCl, $5 \mathrm{mM} \mathrm{KCl,} 1.2 \mathrm{mM} \mathrm{Na}_{2} \mathrm{HPO}_{4}, 10 \mathrm{mM}$ D-glucose, $20 \mathrm{mM}$ Hepes, pH 7.4) during imaging. To perform sptPALM, we used a $405 \mathrm{~nm}$ laser (Stradus 405, Vortran Laser Technology) to photoconvert the mEos3.2-tagged molecules and a $561 \mathrm{~nm}$ laser (Cobolt Jive, Cobolt Lasers) for excitation of the photoconverted molecules. To activate and detect the mEos3.2 signal from the background signals, we used a TIRFM GFP/RFP filter cube (Nikon corporation) in the microscope body, and a T5651pxr long-pass dichroic beam splitter, and an ET600/50m emission filter (Chroma Technology) in the TwinCam (Cairn Research) dual emission image splitter transmission arm. The $405 \mathrm{~nm}$ laser power density was between $3.4 \times 10^{-6}$ and $9.5 \times 10^{-5} \mathrm{~kW} / \mathrm{cm}^{2}$, and the $561 \mathrm{~nm}$ laser power density was set to $\sim 0.14 \mathrm{~kW} / \mathrm{cm}^{2}$. To track Tau ${ }^{\mathrm{WT}}$-HaloTag, we first incubated cells coexpressing Tau ${ }^{\text {WT }}$-HaloTag and the cell fill mEmerald with the Halo ligand $(2 \mathrm{pM})$ for 15 min at $37^{\circ} \mathrm{C}$. Then, cells were washed and Tau ${ }^{\mathrm{WT}}$-HaloTag molecules were tracked using a $642 \mathrm{~nm}$ laser at a power density of $0.12 \mathrm{~kW} / \mathrm{cm}^{2}$, a ZT405/488/561/647rpc quad-band dichroic beam splitter and ZET405/488/561/640m emission filter (Chroma Technology) in the microscope body, and a ZT647rdc dichroic beam splitter and an ET690/50m emission filter (Chroma Technology) in the TwinCam transmission arm.

\section{Nocodazole and $M \beta C D$ treatments}

For nocodazole treatment experiments, cells were incubated with either nocodazole $(5 \mu \mathrm{M})$ or DMSO mixed with culture medium. Cells were then washed and incubated with buffer A containing either nocodazole $(5 \mu \mathrm{M})$ or DMSO during imaging. For $\mathrm{M} \beta \mathrm{CD}$ experiments, cells were washed and incubated with buffer A containing $6 \mathrm{mM} \mathrm{M} \beta C D$. Imaging was performed between 5 and 40 minutes post $\mathrm{M} \beta \mathrm{CD}$ treatment. 


\section{SptPALM analysis}

Tracking. We localized and tracked individual Tau molecules tagged with mEos3.2 as previously described $^{28,41,42}$. Briefly, individual molecules were localized using a wavelet-based segmentation algorithm $^{43}$ and trajectories were computed using a simulated annealing-based tracking algorithm ${ }^{44}$, with the PALM-Tracer tool that operates as a plugin of Metamorph software (Molecular Devices). We used a frame-to-frame particle-linking distance threshold of $318 \mathrm{~nm}$ (3 pixels). We visually inspected for misconnections using the InferenceMAP tool in both fixed and live cells ${ }^{45}$. Of note, we observed a small proportion of mobile molecules in fixed samples, potentially due to incomplete molecular immobilization after fixation using paraformaldehyde ${ }^{46}$.

Intensity and trajectory maps. Intensity and trajectory maps were constructed using detections lasting at least four and eight consecutive frames, respectively. For intensity maps, the local density of each detection was determined by computing the number of detections with a circle of $30 \mathrm{~nm}$ radius using a custom written code.

Mean square displacement and diffusion coefficients. We constructed trajectories of detections that lasted at least eight consecutive frames and computed the MSD of each trajectory. The MSD was fitted by the equation $M S D(\tau)=a+4 D \tau$, where $D$ is the diffusion coefficient, $a$ is the y-intercept and $\tau$ is the time shift. Cells with at least 1,000 trajectories lasting at least 8 frames were considered for analysis with MSD and diffusion coefficients. The average MSD of all trajectories from each analyzed cell was fitted by the equation $\operatorname{MSD}(\tau)=a+4 D_{\text {avg }} \tau$ to estimate the average diffusion coefficient $D_{\text {avg }}$ (Supplementary Fig. 12).

Moment scaling spectrum. Using divide-and-conquer moment scaling spectrum (DC-MSS) tool ${ }^{47}$, we performed the moment scaling spectrum (MSS) analysis to Tau ${ }^{\mathrm{WT}}-\mathrm{mEos} 3.2$ trajectories that lasted at least 20 frames, estimated the slope of MSS, and categorized the trajectories into different motion 
states, as described previously ${ }^{27,47}$. Briefly, for every time shift $\tau$, the moments of displacement, $\mu_{m}$, were computed for $m=1, \ldots, 6$. Next, using the relationship $\mu_{m}(\tau)=4 D_{m} \tau^{\alpha m}$, the generalized diffusion coefficient $D_{m}$ and the exponent $\alpha_{m}$ are estimated for each $m$. The plot $\alpha_{m}$ versus $m$ yields the MSS and the slope of the MSS allows categorizing trajectories into different motion types.

Hidden Markov modelling. The vibrational Bayes SPT (vbSPT) tool ${ }^{48}$ was used to analyze Tau ${ }^{\mathrm{WT}}$ mEos3.2 and Tau ${ }^{\mathrm{S} 262 \mathrm{E} / \mathrm{S} 356 \mathrm{E}}-\mathrm{mE}$ os3 3.2 trajectories. Cells with $\geq 1000$ trajectories were used for this analysis. HMM approach models the trajectories as random transitions between a set of hidden states with different diffusion coefficients. By applying Bayesian model selection to hidden Markov models, the vbSPT analysis infers the number of hidden diffusive states and the associated parameters from the experimental data. Initially, when we allowed a maximum of 10 hidden states, models with 3 or more states provided the best fit (Supplementary Fig. 5). We have therefore presented the parameters estimated by fitting a three-state model to the experimental data (Fig. 11 and Supplementary Figs. 5f, 6j and 11b).

Frame-to-frame displacement analysis. We computed the empirical cumulative frequency distribution of the displacements of Tau molecules tagged with mEos3.2 molecules at 20 ms intervals using the tool ECDF in MATLAB. The one-state model is described by $C(r, \Delta t)=1-f_{1} \exp \left(\frac{-r^{2}}{4 D_{1} \Delta t}\right)$ , the two-state model by $C(r, \Delta t)=1-f_{1} \exp \left(\frac{-r^{2}}{4 D_{1} \Delta t}\right)-f_{2} \exp \left(\frac{-r^{2}}{4 D_{2} \Delta t}\right)$, and the three-state model by $C(r, \Delta t)=1-f_{1} \exp \left(\frac{-r^{2}}{4 D_{1} \Delta t}\right)-f_{2} \exp \left(\frac{-r^{2}}{4 D_{2} \Delta t}\right)-f_{3} \exp \left(\frac{-r^{2}}{4 D_{3} \Delta t}\right)$. Here, $r$ is the displacement, $\Delta t$ is the time interval (20 ms), $D_{1}, D_{2}$ and $D_{3}$ are the diffusion coefficients of the three states, and $f_{1}, f_{2}$ and $f_{3}$ are the state occupancies. We fit the predictions of different models to the data using the non-linear regression tool NLINFIT in MATLAB to estimate the model parameters. 
Voronoï tessellation and time-correlated PALM (tcPALM). We used a cross-correlation-based method to correct for any motion drift during imaging acquisition ${ }^{49}$. We then used the SR-Tesseler tool $^{29}$ to identify the outline of the cell (object). Tau hot spots were identified as regions with local density at least five-fold greater than the average density of the object. Next, we performed the tcPALM analysis ${ }^{30,31}$ by drawing a square region of interest around each spot and computing the number of detections within the region as a function of time. The start and end points of each burst were identified from the cumulative detections using dark time tolerance of 200 frames. Bursts with at least 50 detections were considered for further analysis. To avoid cell edge confounds due to the folding of membranes at the edges, we analyzed cell areas of $\sim 100-400 \mu \mathrm{m}^{2}$, excluding cell edges.

\section{Statistical analysis}

The D'Agostino and Pearson test was used to test for normality, and the Student's $t$-test was used for statistical comparison when the data were normally distributed. When data sets had more than two groups, an ANOVA was used with appropriate corrections for multiple comparisons. Values are represented as the mean \pm s.e.m or mean \pm s.d., as indicated in the figure legends. Data were considered significant at $\mathrm{p}<0.05$. GraphPad Prism 9 was used to perform statistical tests and making figures.

\section{REFERENCES}

1. Polanco, J.C., et al. Amyloid- $\beta$ and tau complexity - towards improved biomarkers and targeted therapies. Nat Rev Neurol 14, 22-39 (2018).

2. Chang, C.W., Shao, E. \& Mucke, L. Tau: Enabler of diverse brain disorders and target of rapidly evolving therapeutic strategies. Science 371 (2021).

3. Brandt, R., Leger, J. \& Lee, G. Interaction of Tau with the neural plasma membrane mediated by Tau's amino-terminal projection domain. J Cell Biol 131, 1327-1340 (1995).

4. Arrasate, M., Pérez, M. \& Avila, J. Tau dephosphorylation at tau-1 site correlates with its association to cell membrane. Neurochem Res 25, 43-50 (2000).

5. Pooler, A.M., et al. Dynamic association of Tau with neuronal membranes is regulated by phosphorylation. Neurobiol Aging 33, 431.e427-438 (2012). 
6. Katsinelos, T., et al. Unconventional secretion mediates the trans-cellular spreading of Tau. Cell Rep 23, 2039-2055 (2018).

7. Merezhko, M., et al. Secretion of Tau via an unconventional non-vesicular mechanism. Cell Rep 25, 2027-2035 e2024 (2018).

8. Elbaum-Garfinkle, S., Ramlall, T. \& Rhoades, E. The role of the lipid bilayer in Tau aggregation. Biophys J 98, 2722-2730 (2010).

9. Patel, N., Ramachandran, S., Azimov, R., Kagan, B.L. \& Lal, R. Ion channel formation by Tau protein: implications for Alzheimer's disease and tauopathies. Biochemistry 54, 7320-7325 (2015).

10. Sallaberry, C.A., et al. Tau and membranes: Interactions that promote folding and condensation. Front Cell Dev Biol 9, 725241 (2021).

11. Padmanabhan, P., Kneynsberg, A. \& Götz, J. Super-resolution microscopy: a closer look at synaptic dysfunction in Alzheimer disease. Nat Rev Neurosci 22, 723-740 (2021).

12. Janning, D., et al. Single-molecule tracking of Tau reveals fast kiss-and-hop interaction with microtubules in living neurons. Mol Biol Cell 25, 3541-3551 (2014).

13. Brandt, R., Trushina, N.I. \& Bakota, L. Much more than a cytoskeletal protein: Physiological and pathological functions of the non-microtubule binding region of Tau. Front Neurol 11, 590059 (2020).

14. Polanco, J.C., Hand, G.R., Briner, A., Li, C. \& Götz, J. Exosomes induce endolysosomal permeabilization as a gateway by which exosomal tau seeds escape into the cytosol. Acta Neuropathol (2021).

15. Georgieva, E.R., Xiao, S., Borbat, P.P., Freed, J.H. \& Eliezer, D. Tau binds to lipid membrane surfaces via short amphipathic helices located in its microtubule-binding repeats. Biophys $J \mathbf{1 0 7}$, 1441-1452 (2014).

16. Mari, S.A., et al. Reversible cation-selective attachment and self-assembly of human Tau on supported brain lipid membranes. Nano Lett 18, 3271-3281 (2018).

17. Magnani, E., et al. Interaction of Tau protein with the dynactin complex. EMBO J 26, 4546-4554 (2007).

18. Gauthier-Kemper, A., et al. The frontotemporal dementia mutation R406W blocks tau's interaction with the membrane in an annexin A2-dependent manner. J Cell Biol 192, 647-661 (2011).

19. Manley, S., et al. High-density mapping of single-molecule trajectories with photoactivated localization microscopy. Nat Methods 5, 155-157 (2008).

20. Mehidi, A., et al. Transient activations of Rac1 at the lamellipodium tip trigger membrane protrusion. Curr Biol 29, 2852-2866 e2855 (2019).

21. Lee, Y., et al. High-throughput, single-particle tracking reveals nested membrane domains that dictate $\operatorname{KRas}(\mathrm{G} 12 \mathrm{D})$ diffusion and trafficking. eLife $\mathbf{8}$ (2019). 
22. Gormal, R.S., et al. Modular transient nanoclustering of activated $\beta 2$-adrenergic receptors revealed by single-molecule tracking of conformation-specific nanobodies. Proc Natl Acad Sci U S A 117, 30476-30487 (2020).

23. Gura Sadovsky, R., Brielle, S., Kaganovich, D. \& England, J.L. Measurement of rapid protein diffusion in the cytoplasm by photo-converted intensity profile expansion. Cell Rep 18, 27952806 (2017).

24. Kühn, T., et al. Protein diffusion in mammalian cell cytoplasm. PLoS One 6, e22962 (2011).

25. Rossier, O., et al. Integrins $\beta 1$ and $\beta 3$ exhibit distinct dynamic nanoscale organizations inside focal adhesions. Nat Cell Biol 14, 1057-1067 (2012).

26. Jacobson, K., Liu, P. \& Lagerholm, B.C. The lateral organization and mobility of plasma membrane components. Cell 177, 806-819 (2019).

27. Jaqaman, K., et al. Cytoskeletal control of CD36 diffusion promotes its receptor and signaling function. Cell 146, 593-606 (2011).

28. Padmanabhan, P., Martinez-Marmol, R., Xia, D., Götz, J. \& Meunier, F.A. Frontotemporal dementia mutant Tau promotes aberrant Fyn nanoclustering in hippocampal dendritic spines. eLife 8, e45040 (2019).

29. Levet, F., et al. SR-Tesseler: a method to segment and quantify localization-based superresolution microscopy data. Nat Methods 12, 1065-1071 (2015).

30. Cisse, II, et al. Real-time dynamics of RNA polymerase II clustering in live human cells. Science 341, 664-667 (2013).

31. Cho, W.K., et al. Mediator and RNA polymerase II clusters associate in transcription-dependent condensates. Science 361, 412-415 (2018).

32. Izeddin, I., et al. Single-molecule tracking in live cells reveals distinct target-search strategies of transcription factors in the nucleus. Elife 3 (2014).

33. Garcia-Parajo, M.F., Cambi, A., Torreno-Pina, J.A., Thompson, N. \& Jacobson, K. Nanoclustering as a dominant feature of plasma membrane organization. J Cell Sci 127, 49955005 (2014).

34. $\mathrm{Xu}, \mathrm{Z}$., et al. Microtubules acquire resistance from mechanical breakage through intralumenal acetylation. Science 356, 328-332 (2017).

35. Chen, Y.M., et al. Microtubule affinity-regulating kinase 2 functions downstream of the PAR3/PAR-6/atypical PKC complex in regulating hippocampal neuronal polarity. Proc Natl Acad Sci U S A 103, 8534-8539 (2006).

36. Zhou, Y. \& Hancock, J.F. Ras nanoclusters: Versatile lipid-based signaling platforms. Biochim Biophys Acta 1853, 841-849 (2015).

37. Orré, T., et al. Molecular motion and tridimensional nanoscale localization of kindlin control integrin activation in focal adhesions. Nat Commun 12, 3104 (2021). 
38. Shibata, A.C., et al. Rac1 recruitment to the archipelago structure of the focal adhesion through the fluid membrane as revealed by single-molecule analysis. Cytoskeleton (Hoboken) 70, 161177 (2013).

39. Padmanabhan, P., et al. Need for speed: Super-resolving the dynamic nanoclustering of syntaxin1 at exocytic fusion sites. Neuropharmacology 169, 107554 (2020).

40. Zhang, M., et al. Rational design of true monomeric and bright photoactivatable fluorescent proteins. Nat Methods 9, 727-729 (2012).

41. Bademosi, A.T., et al. In vivo single-molecule imaging of syntaxin1A reveals polyphosphoinositide- and activity-dependent trapping in presynaptic nanoclusters. Nat Commun 8, 13660 (2017).

42. Joensuu, M., et al. Subdiffractional tracking of internalized molecules reveals heterogeneous motion states of synaptic vesicles. J Cell Biol 215, 277-292 (2016).

43. Izeddin, I., et al. Wavelet analysis for single molecule localization microscopy. Opt Express 20, 2081-2095 (2012).

44. Racine, V., et al. Multiple-target tracking of 3D fluorescent objects based on simulated annealing. IEEE International Symposium on Biomedical Imaging: Macro to nano 1-3, 10201023 (2006).

45. El Beheiry, M., Dahan, M. \& Masson, J.B. InferenceMAP: mapping of single-molecule dynamics with Bayesian inference. Nat Methods 12, 594-595 (2015).

46. Tanaka, K.A., et al. Membrane molecules mobile even after chemical fixation. Nat Methods 7, 865-866 (2010).

47. Vega, A.R., Freeman, S.A., Grinstein, S. \& Jaqaman, K. Multistep track segmentation and motion classification for transient mobility analysis. Biophys $J$ 114, 1018-1025 (2018).

48. Persson, F., Lindén, M., Unoson, C. \& Elf, J. Extracting intracellular diffusive states and transition rates from single-molecule tracking data. Nat Methods 10, 265-269 (2013).

49. Andronov, L., Lutz, Y., Vonesch, J.L. \& Klaholz, B.P. SharpViSu: integrated analysis and segmentation of super-resolution microscopy data. Bioinformatics 32, 2239-2241 (2016). 